\title{
A long surviving case of resected gastric cancer presenting with a synchronous adrenal metastasis
}

\author{
Takeo Kosaka, Nobuo Ueshige, Jun-ichi Sugaya, Yasuharu Nakano, Fujio Tomita, Hitoshi Saito, \\ ICHIRo KITA, and SHIGEKI TAKASHIMA \\ Department of Surgery II, Kanazawa Medical University, 1-1, Daigaku, Uchinada-machi, Kahoku-gun, Ishikawa 920-0293, Japan
}

\begin{abstract}
:
Clinically curable adrenal metastases are rare. We treated a patient with gastric cancer and a synchronous adrenal metastasis who underwent curative resection. Upper GI examinations of a 75-year-old man revealed a Borrmann 3 gastric tumor in the proximal stomach. CT indicated a giant gastric tumor that invaded the pancreatic tail, and the left adrenal gland seemed normal. He was subjected to a total gastrectomy and a distal pancreatosplenectomy. Because a mass was palpated intraoperatively in the left adrenal gland, it was also removed. The gastric tumor was histopathologically a poorly differentiated adenocarcinoma with scirrhous invasion that invaded the pancreas, and the histopathological findings of the left adrenal tumor were compatible with those of gastric cancer. At present, 6 years after the operation, there has been no clear sign of cancer recurrence. It may be rational to excise the left adrenal gland en bloc in patients with serosa-positive Borrmann 3-4 gastric cancer.
\end{abstract}

Key words: gastric cancer, synchronous adrenal metastasis, surgical resection

\section{Introduction}

Metastases to the adrenal glands are frequently found at autopsy, but clinically curable adrenal metastases are rare. We treated a patient with gastric cancer and a synchronous adrenal metastasis who underwent curative resection.

Offprint requests to: $\mathrm{T}$. Kosaka

Received for publication on Jan. 28, 1999; accepted on May 29, 1999

\section{Case report}

The patient, a 75-year-old man, consulted us because of epigastric discomfort in February 1993. Upper GI series and gastroscopy revealed a Borrmann 3 gastric tumor in the proximal stomach. Laboratory data showed slight anemia, $10.5 \mathrm{~g} / \mathrm{dl}$ of hemoglobin, and elevation of tumor markers $(174 \mathrm{ng} / \mathrm{ml}$ of CA $19-9$ and $298 \mathrm{ng} / \mathrm{ml}$ of Sialyl $\mathrm{Tn})$. Computed tomography (CT) indicated a giant gastric tumor that invaded the pancreatic tail; the left adrenal gland seemed normal (Fig. 1). He was subjected to a total gastrectomy and a distal pancreatosplenectomy. Although the left adrenal gland was within normal limits in size, it was intraoperatively palpated as a hard mass. Therefore it was also removed during an abdominal paraaortic lymph node dissection.

The postoperative course was uneventful. Histopathological examinations revealed that the gastric tumor, which measured $8.7 \times 8.5 \mathrm{~cm}$, was predominantly a poorly differentiated adenocarcinoma with scirrhous invasion with a partial component of tubular adenocarcinoma that invaded the pancreas and involved nine lymph nodes [perigastric: \#1, \#2, \#3, \#4s, and \#4d; along splenic artery: \#11(n2)]. The histopathological findings of the left adrenal tumor revealed that there were adenocarcinoma cells nested within the normal adrenal gland and that this was compatible with those of gastric cancer (Fig. 2). At present, 6 years after the operation, the patient is still alive with no evidence of recurrence of cancer.

\section{Discussion}

Adrenal metastases occur most commonly in patients with kidney, lung, and colon carcinomas. Recently, there have been three articles on adrenalectomy for metastatic carcinomas. Lo et al. [1] performed adrenalectomy in 52 patients with metastatic adrenal carci- 


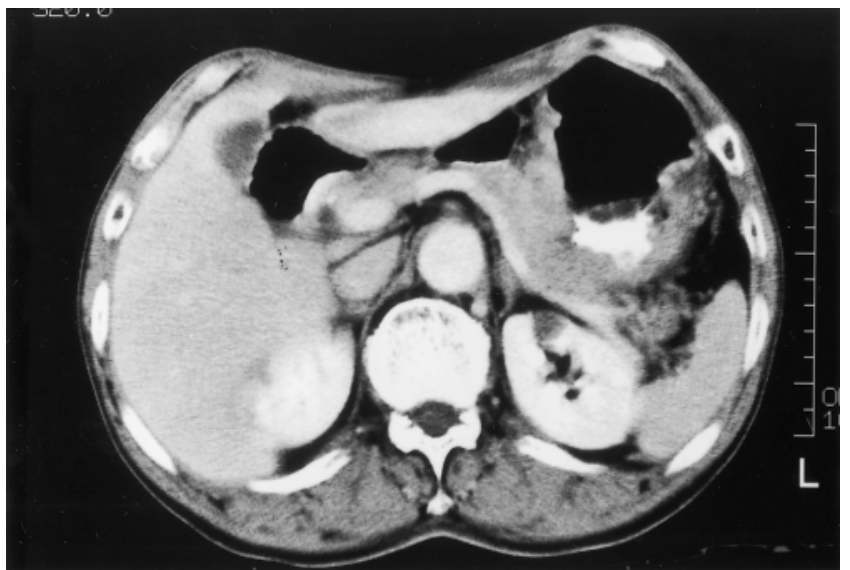

Fig. 1. Enhanced abdominal CT scan reveals a giant gastric tumor that invades the pancreatic tail. The left adrenal gland, visible attached to the left kidney, is within normal size limits

noma; the primary tumor site was the kidney in 15 , the lung in 11, and the colon in 7. Kim et al. [2] also reported that, of their 37 patients subjected to adrenalectomy for metastatic cancer, the primary tumor had been located in the lung in 17 patients, the kidney in 9 , and the colon in 5. Furthermore, Wade et al. [3], from the database of 159 hospitals, evaluated 49 cases of adrenalectomy for metastasis; among them, the primary tumor had been located in the lung in 14 patients, the kidney in 12 , and the colon in 8 . However, for adrenal metastasis of gastric carcinoma origin, Lo et al. [1] experienced only 3 cases in which this had metastasized from a tumor in the stomach, but the other two study groups reported no such cases. It is assumed that an adrenal metastasis from stomach carcinoma is rarely resectable, especially in a curable manner. The patient's prognosis after adrenalectomy for a metastatic tumor is poor, as the overall 5year survival rate was reported to be $13 \%-24 \%$ and the median survival time 13-21 months [1-3].

Concerning the rate of adrenal metastasis from gastric cancer, $16 \%-18 \%$ of patients with gastric cancer show adrenal involvement at autopsy [4,5]. In advanced cases, adrenal metastasis from gastric cancer is not rare. On the other hand, Furukawa et al. [6] reported that, in $3(5.5 \%)$ of 54 surgically resected cases with Borrmann 4 gastric cancer, the left adrenal gland was involved. Such involvement is considered to occur in the manner of direct invasion or by lymphangitic routes. As for the metachronous metastases to the adrenal gland from gastric cancer, there have been a few resected cases [5,7-10].

Metastases from a gastric cancer to the adrenal glands have been supposed to occur by direct invasion, via a hematogenous route, or via a lymphangitic route. No

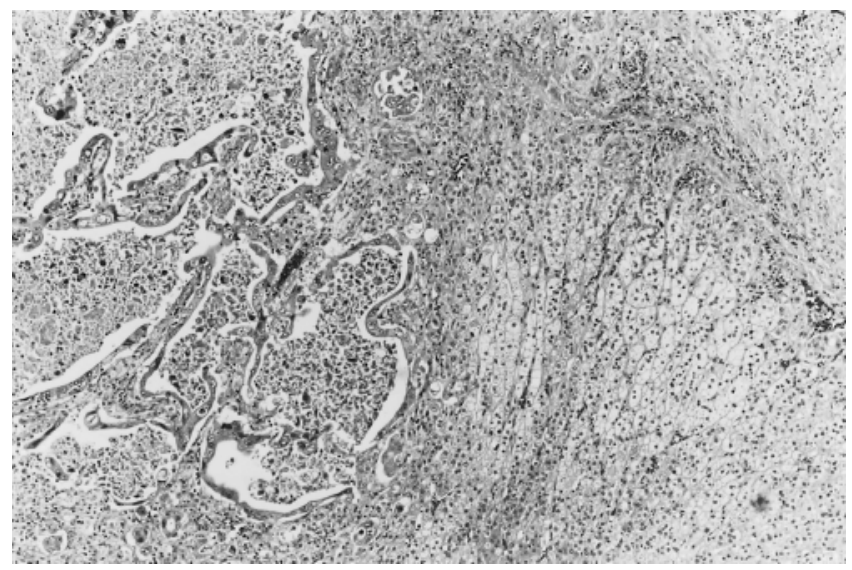

Fig. 2. Cross section through the adrenal tumor. Microscopic examination revealed that the cancer nest (left side), which consisted of tubular adenocarcinoma cells, had invaded the adrenal gland (right side). $\mathrm{H} \& \mathrm{E} . \times 20$

direct invasion was observed in this case, either macroscopically or microscopically. Because histopathological examinations revealed an invasive cancer nest around the adrenal gland as well as within it, it is conceivable that the tumor metastasized via a lymphangitic route.

Extended gastrectomy for advanced cancer is associated with a high morbidity rate. Also, adrenalectomy is considered to involve acute adrenal insufficiency when the contralateral adrenal gland is not functioning properly. However, it may be rational to excise the left adrenal gland en bloc in patients with serosa-positive Borrmann 3-4 gastric cancer, as in the current case.

\section{References}

1. Lo CY, van Heerden JA, Soreide JA, Grant CS, Thompson GB, Lloyd RV, et al. Adrenalectomy for metastatic disease to the adrenal glands. Br J Surg 1996;83:528-31.

2. Kim SH, Brennan MF, Russo P, Burt ME, Coit DG. The role of surgery in the treatment of clinically isolated adrenal metastasis. Cancer (Phila) 1998;82:389-94.

3. Wade TP, Longo WE, Virgo KS, Johnson FE. A comparison of adrenalectomy with other resection for metastatic cancers. Am J Surg 1998;175:183-6.

4. Cedermark BJ, Blumenson LE, Pickren JW, Elias EG. The significance of metastases to the adrenal gland from carcinoma of the stomach and esophagus. Surg Gynecol Obstet 1977;145:418.

5. Yoshizumi Y, Shima S, Sugiura Y, Yonekawa H, Morisaki Y, Tanaka S. A case of resected metastatic adrenal carcinoma from gastric cancer. Gann No Rinsho 1989;35:1699-704.

6. Furukawa $\mathrm{H}$, Hiratsuka $\mathrm{M}$, Iwanaga $\mathrm{T}$, Imaoka $\mathrm{S}$, Ishikawa $\mathrm{O}$, Kabuto T, et al. Extended surgery - left upper abdominal exenteration plus Appleby's method - for type 4 gastric carcinoma. Ann Surg Oncol 1997;4:209-14.

7. Kamei $T$, Hasegawa $H$, Ogiso $S$, Murata $T$, Nagasawa $K$, Taniai H. A case of a surgically treated patient with an 
other organ metastasis (adrenal gland, liver) of gastric cancer, with a favorable prognosis. Nippon Rinshogekaigakkai Zasshi 1997;58:815-19.

8. Ohshima T, Noguchi Y, Doi C, Yoshikawa T, Makino T, Amano $\mathrm{T}$, et al. A solitary metachronous metastasis to the right adrenal gland from gastric cancer. Geka 1997;59:867-70.
9. Aoki F, Kitamura M, Arai K, Miyashita K, Iwasaki Y. A case of resected gastric cancer with synchronous huge adrenal metastasis. Nippon Shoukakigekagakkai Zasshi 1995;28:1081-5.

10. Toochika H, Hirata K, Nakao S, Ishii T, Eida K, Takahara O. A resected case of gastric cancer metastasized to the adrenal gland. Nippon Rinshogekagakkai Zasshi 1993;54:3147-50. 